ORIGINAL ARTICLE

\title{
Tumour angiogenic activity and vascular survival ability in bladder carcinoma
}

\author{
I Papadopoulos, A Giatromanolaki, M I Koukourakis, E Sivridis
}

J Clin Pathol 2004;57:250-255. doi: 10.1136/jcp.2003.012005

See end of article for authors' affiliations .....................

Correspondence to: Professor I Papadopoulos, Department of Urology, Democritus University of Thrace, Alexandroupolis 68100, Greece; escrrid@med.duth.gr

Accepted for publication 10 September 2003
Background: Tumour angiogenic activity (TAA) is an important prognostic factor in many human tumours, including transitional cell carcinomas of the urinary bladder. The new tumour vessels are formed in the invading tumour front. This peripheral tumour area is internalised as soon as the growing tumour forms a new front.

Aims: To investigate and compare TAA with the ability of the tumour vasculature to survive (VSA) in inner tumour areas.

Methods: Fifty one cystectomy specimens with transitional cell carcinoma of the urinary bladder were studied. Sections were stained immunohistochemically for endothelial cells and proliferation activity, using the monoclonal antibodies CD31 and MIB-1, respectively. TAA was studied at the invading tumour edgedesignated as the mean number of blood vessels in three "hot spots" at this site. VSA was assessed by comparing the vascular density in peripheral and inner tumour areas.

Results: High TAA at the invading tumour edge significantly correlated with lymph node involvement, but not with patient survival. Extensive lymphocytic infiltration was more frequent in tumours with high TAA. VSA was significantly higher in tumours of high proliferation index, high histological grade, advanced T stage, and poor prognosis. However, there was no association with metastasis to regional lymph nodes. Conclusion: VSA and TAA provide a more complete profile of the tumour vasculature and are associated with aggressive tumour behaviour in transitional cell carcinomas of the urinary bladder. The qualitative information provided by VSA may be important for the identification of angiogenic tumours with differential responses to various antiangiogenic treatments.
A high vascular density, expressed as tumour angiogenic activity (TAA), is essential for tumour growth and invasion. ${ }^{1}$ The newly formed vessels, derived from preexisting capillaries at the tumour-normal tissue interface, supply the developing tumour with additional oxygen and glucose, and facilitate metastasis because the immature and deficient capillary vessel wall is easily permeable to invading tumour cells. ${ }^{2}$ Therefore, it has been suggested that an increased TAA characterises tumours with an aggressive clinical behaviour. This has been repeatedly confirmed by a plethora of clinicopathological studies on the postoperative outcome of patients with cancer. ${ }^{3}$

"We evaluated the importance of vascular survival ability, relative to tumour angiogenic activity, in a series of transitional cell carcinomas of the urinary bladder treated with cystectomy"

As malignant tumours grow, the new vasculature at the invading tumour front is gradually incorporated into the main tumour, becoming part of the inner tumour area, and thus facing the unfavourable intratumorous conditions of hypoxia and acidity, while new invading edges appear. ${ }^{4}$ At this site, the balance of proliferation versus apoptosis shifts towards apoptosis and the survival of blood vessels becomes problematic. Thompson et al in 1987 were the first to point out the dramatic decrease in vascular density in the inner tumour areas compared with peripheral areas when studying experimental breast carcinomas, ${ }^{5}$ an observation that was subsequently confirmed in human tissues. ${ }^{6}$ The ability of tumours to maintain a high vascular density in their inner masses relative to the invading tumour fronts varies between tumours, and this feature has been designated the vascular survival ability (VSA). ${ }^{7}$ We described a simple and effective method for assessing VSA in paraffin wax embedded sections, based on three consecutive recordings of the vascular density along an imaginary line connecting the invading tumour front and the heart of the tumour. VSA emerged as an important vascular parameter, bearing independent prognostic information in a variety of tumours, including lung, colon, and breast. ${ }^{7-9}$

In our present study, we evaluated the importance of VSA, relative to TAA, in a series of transitional cell carcinomas of the urinary bladder treated with cystectomy. Invading transitional cell cancer of the urinary bladder arises from early exophytic superficial lesions growing around a vasculoconnective core. Their subsequent transition to carcinomas invading into the muscularis is associated with changing patterns of neoangiogenesis, where newly formed vessels invade the muscle layer together with the cancer cells. Therefore, studying the patterns of angiogenesis and of vascular survival is of particular interest in this tumour type. Correlations with histopathological and tumour growth variables, and with patients prognosis, were also sought.

\section{MATERIALS AND METHODS}

Formalin fixed, paraffin wax embedded tissues from 51 patients with invasive transitional cell carcinoma of the urinary bladder, treated with surgery alone were retrieved. Haematoxylin and eosin stained sections were used to select

Abbreviations: MVD, microvessel density; TAA, tumour angiogenic activity; PI, proliferation index; VD, vascular density; VSA, vascular survival ability 


\begin{tabular}{|ll|}
\hline Table 1 Patients and disease characteristics \\
\hline Patient characteristics (N=51) \\
\hline Median age (range) & $65(27-76)$ \\
Sex (male/female) & $43 / 8$ \\
Performance status WHO 0,1 & 51 \\
Histology: transitional cell carcinoma & 51 \\
T stage & 8 \\
T1 & 10 \\
T2 & 29 \\
T3 & 4 \\
T4 & 31 \\
Regional nodes & 20 \\
Negative & \\
Positive & 5 \\
Grade & 27 \\
2 & 19 \\
3 & 51 \\
4 & \\
Previous chemo/radiotherapy: none & \\
\hline WHO, World health Organisation. & \\
\hline
\end{tabular}

tissue sections containing both invading and inner tumour areas for our immunohistochemical study.

Table 1 provides details of the patients and their disease characteristics. All patients in the series were treated with radical cystectomy, whereas those who received adjuvant chemotherapy or radiotherapy were excluded from our study to avoid bias relevant to tumour chemosensitivity or radiosensitivity. Patients who died within 30 days of surgery were also excluded to avoid bias from perioperative mortality. At the time of analysis, 12 of 51 patients were suffering from disease. The follow up of patients ranged from four to 60 months (median, 29), whereas for patients who were still alive it ranged from 18 to 60 months (median, 39).

\section{Immunohistochemical staining}

The JC70 monoclonal antibody (Dako, Glostrup, Denmark), which recognises CD31 (platelet/endothelial cell adhesion molecule; PECAM-1), was used for microvessel staining on $3 \mu \mathrm{m}$ thick sections. Sections were dewaxed, rehydrated, and predigested with protease type XXIV for 20 minutes at $37^{\circ} \mathrm{C}$. A standard streptavidin-biotin immunoperoxidase method was used. Endogenous peroxidase activity was blocked by exposure to absolute methanol containing 3\% hydrogen peroxide. Antigen retrieval was achieved by microwave heating. Non-specific background staining was reduced by a protein blocking agent. The primary antibodies were applied for 75 minutes at room temperature. The sections were then sequentially incubated with: (1) rabbit antimouse antibody (Kwik biotinylated secondary; Immunon, Shandon, Pittsburgh, Pennsylvania, USA) for 15 minutes, and (2) Kwik streptavidin peroxidase reagent (Immunon, Shandon) for 15 minutes. Antibody reactivity was detected with 3,3'-diaminobenzidine as chromogen. The slides were counterstained with Mayer's haematoxylin. Known positive controls were included in each staining run. Omission of the primary antibody and replacement by normal rabbit serum at the same concentration was used as a negative control.

\section{Assessment of TAA and VSA}

Three areas of highest vascularisation ("hot spots") at the boundary of the tumour and normal bladder tissue were assessed for each case. The number of vessels in each $\times 200$ magnification optical field was counted. The mean value of these areas (tl areas) - that is, the vascular density (VD) was designated as the TAA.

Subsequently, vessel counting was performed in four consecutive $\times 200$ magnification fields, starting from the normal bladder ( $\mathrm{n}$ field) and moving towards the central areas of the tumour-that is, from the invading tumour edge/ tumour periphery ( $\mathrm{tl}$ field), through the intermediate inner tumour area (t2 field), to a more inner one ( $\mathrm{t} 3$ field). Each optical field was estimated to have a $2 \mathrm{~mm}$ diameter. Using the scores obtained from counting the $\mathrm{n}, \mathrm{t} \mathrm{l}, \mathrm{t} 2$ and $\mathrm{t} 3$ areas, the mean VD in these areas was assessed. Cases were divided into two groups, according to their ability to maintain the VD in the inner tumour areas. Cases with a mean $\mathrm{t} 2+\mathrm{t} 3 \mathrm{VD}$ higher than $50 \%$ of the $t 1$ VD were considered to have a high VSA.

Assessment of the "edvin" types of vascularisation Combining the TAA and VSA gives four different patterns of vascularisation, which can be described by the "edvin" score (edge $v$ inner), as reported previously. ${ }^{7-9}$ Thus, tumours were categorised as follows: edvin type 1 tumours had low TAA/ VSA; edvin type 2 tumours had low TAA/high VSA; edvin type 3 tumours had high TAA/low VSA; and edvin type 4 tumours had high TAA/VSA. In the first study reported by our group on the edvin score in non-small cell lung cancer, ${ }^{7}$ type 1 and 2 tumours were combined in one group of low vascularisation (reported as edvin 1), whereas only highly angiogenic tumours (high TAA) were divided in two groups of low and high VSA (reported as edvin 2 and 3, respectively). In subsequent studies, ${ }^{89}$ we decided to group tumours with low TAA according to their VSA because this allows the identification of an additional group of tumours with low TAA but with the ability to sustain the survival of the neovasculature once incorporated into the tumour body. Whether such a subgroup has a different biological behaviour is unknown. In that way, four edvin types are recognised for analysis.

\section{Assessment of the proliferation index}

The proliferation index (PI) was assessed using the MIB-1 monoclonal antibody (clone PRO224; YLEM, Rome, Italy). Antigen retrieval was achieved by microwaving the samples $(3 \times 5$ minutes $)$. A standard streptavidin-biotin immunoperoxidase method was used, as described for JC70 immunostaining. The primary antibody (at a $1 / 30$ dilution) was applied overnight at room temperature. The percentage of cells with nuclear reactivity was recorded in all fields, and the mean value was calculated. The median MIB-1 score was used as a cutoff point to define groups of tumours with a low (lower than the median) or high PI.

\section{Assessment of lymphocytic infiltration and necrosis}

The degree of lymphocytic infiltration was assessed on haematoxylin and eosin sections by two pathologists. An intense lymphocytic infiltration in more than $50 \%$ of the optical fields examined (excluding areas of necrosis) was considered to be high (as opposed to low). Similarly, the presence of areas of necrotic tissue in more than $50 \%$ of the optical fields characterised samples as bearing extensive necrosis (as opposed to limited and/or absent necrosis).

\section{Statistical analysis}

Statistical analysis was carried out and graphs were plotted using the GraphPad Prism 2.01 and the GraphPad Instat packages. Survival curves were plotted using the method of Kaplan and Meier, and the log rank test was used to determine significant differences between life tables. The Fisher's exact test was used for testing relations between categorical tumour variables. A Cox proportional hazard model was used to assess the effects of patient and tumour variables on overall survival. All statistical tests used were two sided. A p value $<0.05$ was required for significance. 


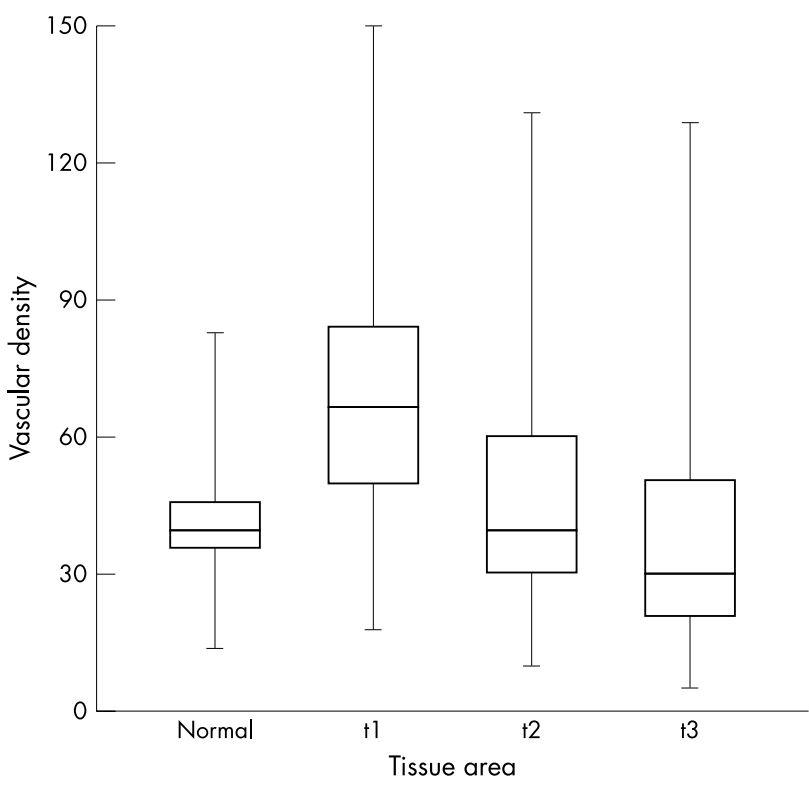

\section{RESULTS}

Tumour angiogenic activity

The median VD was 67 vessels for each $\times 200$ magnification optical field (range, 18-210) at the invading tumour front ( $\mathrm{tl}$ area), and 40 vessels for each $\times 200$ magnification optical field (range, 14-83) in normal bladder tissue. This difference was significant (fig 1 ). Using the median VD value of the $\mathrm{tl}$ area $(<67 v \geqslant 67)$, we defined two groups of tumours, those with low (25 points) and with high (26 points) TAA.

The median MIB-1 PI was 5\% (range, 0-70\%). Using this cutoff point, 35 tumours were of high $(\geqslant 5 \%)$ and 16 of low $(<5 \%)$ PI. A high TAA was associated with nodal involvement $(\mathrm{p}=0.04)$, but not with $\mathrm{T}$ stage, histological grade, or PI (tables 2 and 3). High PI was also linked to advanced T stage $(\mathrm{p}=0.03)$. Using $10 \%$ as a cutoff point for PI (as suggested in many published studies), no association of PI with the examined variables was noted.

\section{Vascular survival ability}

The median VD value was 40 vessels for each $\times 200$ magnification optical field (range, 10-131) in the t2 area, and 30 vessels for each $\times 200$ magnification optical field (range, 5-129) in the $t 3$ area $(p<0.0001$; fig 1). Given that the median VD obtained at the invading tumour front (tl

\begin{tabular}{|c|c|c|}
\hline Normal $v+1$; & $p<0.0001$ & Figure 1 Vascular density in normal \\
\hline Normal $v \nmid 2$ & $p=032$ & $\begin{array}{l}\text { bladder, the invading fumour edge } \\
\text { and inner tumour areas ( }+2 \text { and }+3 \text { ) }\end{array}$ \\
\hline Normal $v+3$; & $p=0.44$ & \\
\hline$+1 v+2,+3$ & $p<0.0001$ & \\
\hline$+2 v+3$ & $p=0.10$ & \\
\hline
\end{tabular}

Normal $v+1 ; \quad p<0.0001$ Figure 1 Vascular density in normal

$+2 v+3$;

area) was significantly higher (67 vessels for each $\times 200$ magnification optical field, with a range 18-210), it is obvious that, overall, the VD was decreasing gradually from the invading tumour edge towards the inner tumour areas.

Of the 51 cases that comprised our study, 32 had a mean $\mathrm{VD}$ in the $\mathrm{t} 2 / \mathrm{t} 3$ areas that was more than $50 \%$ of the VD recorded in the $\mathrm{tl}$ area. These cases were considered to have a high VSA, whereas the remaining 19 were considered to have a low VSA. Figure 2 shows bladder carcinomas with high versus low VSA. A high VSA was significantly associated with high PI $(\mathrm{p}=0.01)$, advanced $\mathrm{T}$ stage $(\mathrm{p}=0.04)$, and grade 4 lesions $(p=0.01)$ (tables 2 and 3$)$.

\section{Edvin types of bladder cancer vascularisation}

By combining TAA and VSA, the 51 cases of transitional cell carcinoma of the bladder were grouped into four different types of vascularisation expressed by the edvin score. No association between TAA and VSA was noted (table 3). Edvin 1 tumours were the only type associated with absence of lymph node involvement, whereas edvin type 4 was linked to advanced $\mathrm{T}$ stage of disease. Extensive necrosis was more frequently noted in edvin 1 type tumours, although this difference was not significant (four of nine versus six of 41; $\mathrm{p}=0.11)$.

Table 2 Associations of MIB-1 proliferation index, vascular survival ability (VSA), and tumour angiogenic activity (TAA) with stage, histological grade, and extent of lymphocytic infiltration (LI)

\begin{tabular}{|c|c|c|c|c|c|c|c|c|c|}
\hline & \multicolumn{3}{|c|}{ MIB-1 } & \multicolumn{3}{|l|}{ VSA } & \multicolumn{3}{|l|}{ TAA } \\
\hline & Low & High & $p$ Value & Low & High & $\mathrm{p}$ Value & Low & High & $\mathrm{p}$ Value \\
\hline \multicolumn{10}{|l|}{$\mathrm{T}$ stage } \\
\hline 2 & 9 & 9 & \multirow{3}{*}{0.03} & 10 & 8 & \multirow{3}{*}{0.04} & 9 & 9 & \\
\hline 3 & 7 & 22 & & 9 & 20 & & 15 & 14 & \multirow[t]{2}{*}{0.91} \\
\hline 4 & 0 & 4 & & 0 & 4 & & 1 & 3 & \\
\hline \multicolumn{10}{|l|}{$\mathrm{N}$ stage } \\
\hline 0 & 11 & 20 & \multirow[t]{2}{*}{0.54} & 13 & 18 & \multirow[t]{2}{*}{0.76} & 19 & 12 & \multirow[t]{2}{*}{0.04} \\
\hline 1 & 5 & 15 & & 6 & 14 & & 6 & 14 & \\
\hline \multicolumn{10}{|l|}{ Grade } \\
\hline $2 / 3$ & 12 & 20 & \multirow[t]{2}{*}{0.22} & 16 & 16 & \multirow[t]{2}{*}{0.01} & 17 & 15 & \multirow[t]{2}{*}{0.44} \\
\hline 4 & 4 & 15 & & 3 & 16 & & 8 & 11 & \\
\hline \multicolumn{10}{|l|}{ LI } \\
\hline Low & 13 & 3 & \multirow[t]{2}{*}{0.20} & 13 & 6 & \multirow[t]{2}{*}{0.99} & 19 & 6 & \multirow[t]{2}{*}{0.23} \\
\hline High & 21 & 14 & & 21 & 11 & & 15 & 11 & \\
\hline
\end{tabular}

The cutoff point for MIB- 1 was $5 \%$ of stained nuclei; the cutoff point for TAA was 67 vessels for each $\times 200$ magnification optical field. 


\begin{tabular}{|c|c|c|c|c|c|c|}
\hline & \multicolumn{3}{|c|}{ MIB-1 } & \multicolumn{3}{|c|}{ VSA } \\
\hline & Low & High & $\mathrm{p}$ Value & Low & High & p Value \\
\hline \multicolumn{7}{|l|}{ TAA } \\
\hline Low & 7 & 18 & 0.61 & 9 & 16 & 0.99 \\
\hline High & 9 & 17 & & 10 & 16 & \\
\hline \multicolumn{7}{|l|}{ VSA } \\
\hline Low & 10 & 9 & 0.01 & - & - & - \\
\hline High & 6 & 26 & & - & - & \\
\hline
\end{tabular}

\section{Lymphocytic infiltration versus angiogenesis variables}

High lymphocytic infiltration of the tumorous stroma was more frequent in tumours with high TAA (11 of $17 v 15$ of $34)$, but the difference was not significant $(p=0.23)$. Similarly, we found no association between the extent of lymphocytic infiltration and VSA or edvin types. Analysis of nodal involvement, according to TAA and lymphocytic infiltration, revealed that tumours with high TAA migrated to the lymph nodes considerably more frequently in the absence of an immune response (table 4).

\section{Survival analysis}

Survival analysis according to Kaplan-Meier showed that $\mathrm{N}$ stage was the best predictor of survival $(p=0.003)$, followed by T stage, histological grade, MIB-1 PI, and VSA $(\mathrm{p}=0.03$; fig 3$)$. No association of TAA with prognosis was noted, although highly angiogenic tumours showed a trend for a worse outcome in the absence of lymphocytic infiltration (fig 4). In multivariate analysis, $\mathrm{N}$ stage was the only factor that approached independent significance (table 5).

\section{DISCUSSION}

The role of angiogenesis and angiogenic stimulating factors in tumour growth and metastasis has been investigated
Table 4 Combined analysis of TAA and lymphocytic infiltration (LI) in relation to the incidence of node involvement

\begin{tabular}{llll}
\hline & \multicolumn{3}{l}{ Node involvement } \\
\cline { 2 - 4 } & No & Yes & p Value \\
\hline TAA/LI & 6 & 9 & \\
High/low & 8 & 2 & 0.08 \\
High/high & 17 & 8 & \\
Low/low & & & \\
\hline
\end{tabular}

extensively over the past few years. ${ }^{10}$ The first clinicopathological study examining the prognostic role of intratumorous microvessel density (MVD) in patients with transitional cell carcinoma of the urinary bladder was reported in 1994 by the Oxford angiogenesis group. ${ }^{11}$ Despite the small number of patients, the authors concluded that an increased MVD was as informative as the stage of disease in predicting overall survival. In a subsequent larger study from the University of Southern California, a high MVD was associated with spread of disease to lymph nodes and poor disease specific survival. ${ }^{12}$
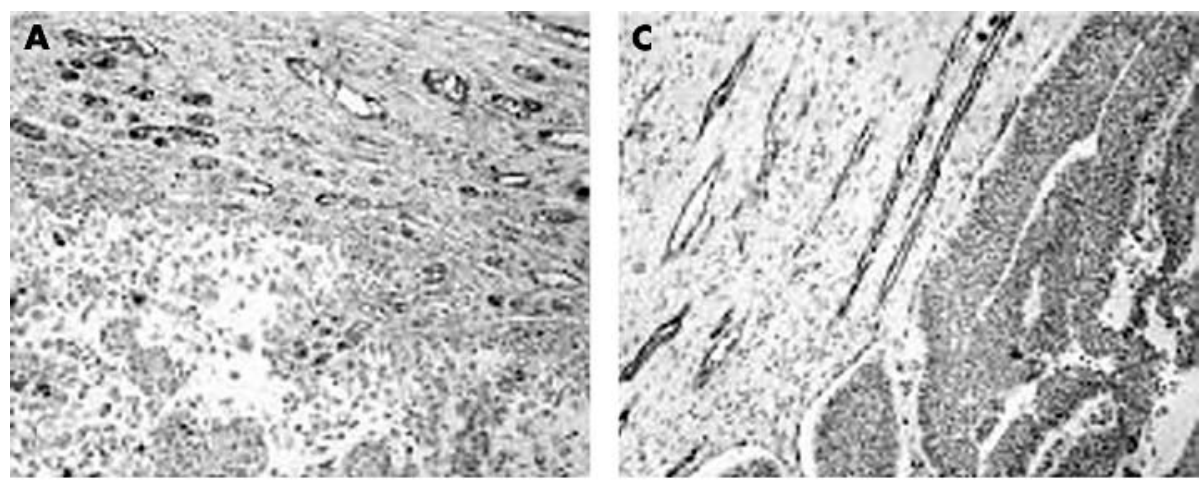

Figure 2 CD31 immunohistochemical images of a highly angiogenic bladder cancer with (A) high vascular density tumour angiogenic activity (TAA) in the invading front and (B) high vascular survival ability (high vascular density in inner +3 tumour areas). (C) A bladder carcinoma with high angiogenic activity (TAA) in the invading tumour edge, but (D) dramatically reduced vascular density in the inner areas ( +3 area).
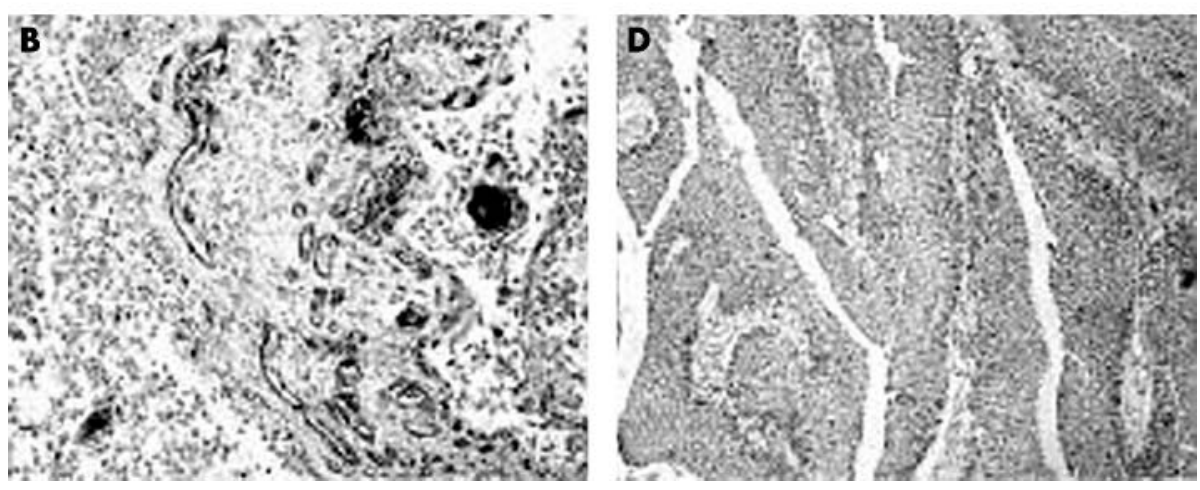


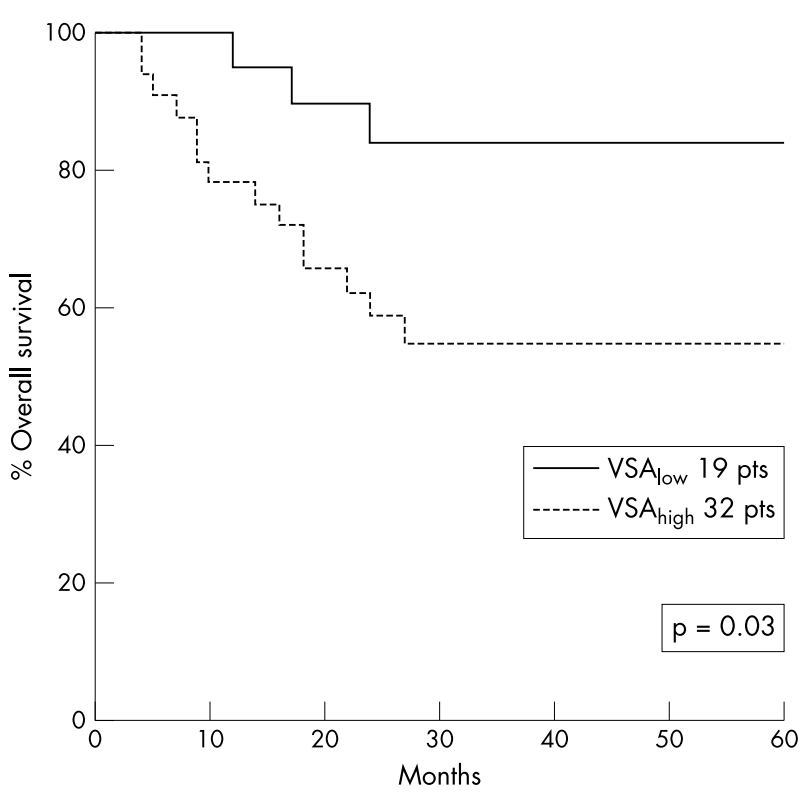

Figure 3 Kaplan-Meier survival curves stratified for vascular survival ability (VSA).

Thereafter, several studies confirmed the ominous prognostic significance of increased MVD in both transitional ${ }^{13-16}$ and squamous cell carcinomas of the urinary bladder. ${ }^{17}$ In sharp contrast to the above studies, a recent report from the Danish Cancer Institute linked high intratumorous MVD with a better disease specific survival for patients with bladder carcinoma. $^{18}$ The authors also noted a direct correlation between high MVD and intense inflammatory cell infiltration of the tumour. Another study by Sagol et al also failed to demonstrate an association between MVD and the recurrence of bladder carcinoma. ${ }^{19}$

In our study, a high vascular density at the invading tumour front (high TAA) was significantly associated with lymph node involvement, but a similar trend was not
Table 5 Univariate (log rank) and multivariate survival analyses

\begin{tabular}{llll}
\hline & & \multicolumn{2}{l}{ Multivariate } \\
\cline { 3 - 4 } & Univariate p value & p Value & † Ratio \\
\hline T stage & 0.01 & 0.56 & 0.57 \\
N stage & 0.003 & 0.08 & 1.88 \\
Grade & 0.01 & 0.61 & 0.50 \\
MIB1 & 0.03 & 0.22 & 1.23 \\
TAA & 0.72 & 0.97 & 0.03 \\
VSA & 0.03 & 0.35 & 0.92 \\
\hline \multirow{2}{*}{ TAA, tumour angiogenic activity; VSA, vascular survival ability. }
\end{tabular}

confirmed for patient survival. Extensive lymphocytic infiltration was more frequent in tumours with high TAA, as suggested by Offersen et al, ${ }^{18}$ but such differences did not reach significance, perhaps because of the low number of cases in our study. Furthermore, tumours with high TAA metastasised to lymph nodes most often in the absence of lymphocytic infiltration, suggesting a role for the immune response in the control of metastatic spread. However, the presence of an intense lymphocytic response may not only suppress the metastatic potential of bladder carcinoma but may also facilitate its angiogenic process. This relation between high angiogenesis and intense lymphocytic infiltration was in accord with previous studies on lung and colon carcinomas. $^{2021}$ Highly angiogenic tumours were associated with poor prognosis only in the presence of a sparse lymphocytic response.

Our results with regard to VSA were also interesting. Thus, in agreement with previously reported studies on lung, colon, and breast cancer, ${ }^{7-9}$ the VD in transitional cell carcinomas of the urinary bladder was significantly higher at the invading tumour edge than in the inner tumour areas, showing a gradual drop towards the central areas of the tumour. At these sites, the ability of bladder carcinomas to maintain a relatively high VD varied considerably between tumours. Thus, the VSA was significantly higher in tumours of high histological grade and advanced $\mathrm{T}$ stages of disease, but showed no association with nodal involvement. These

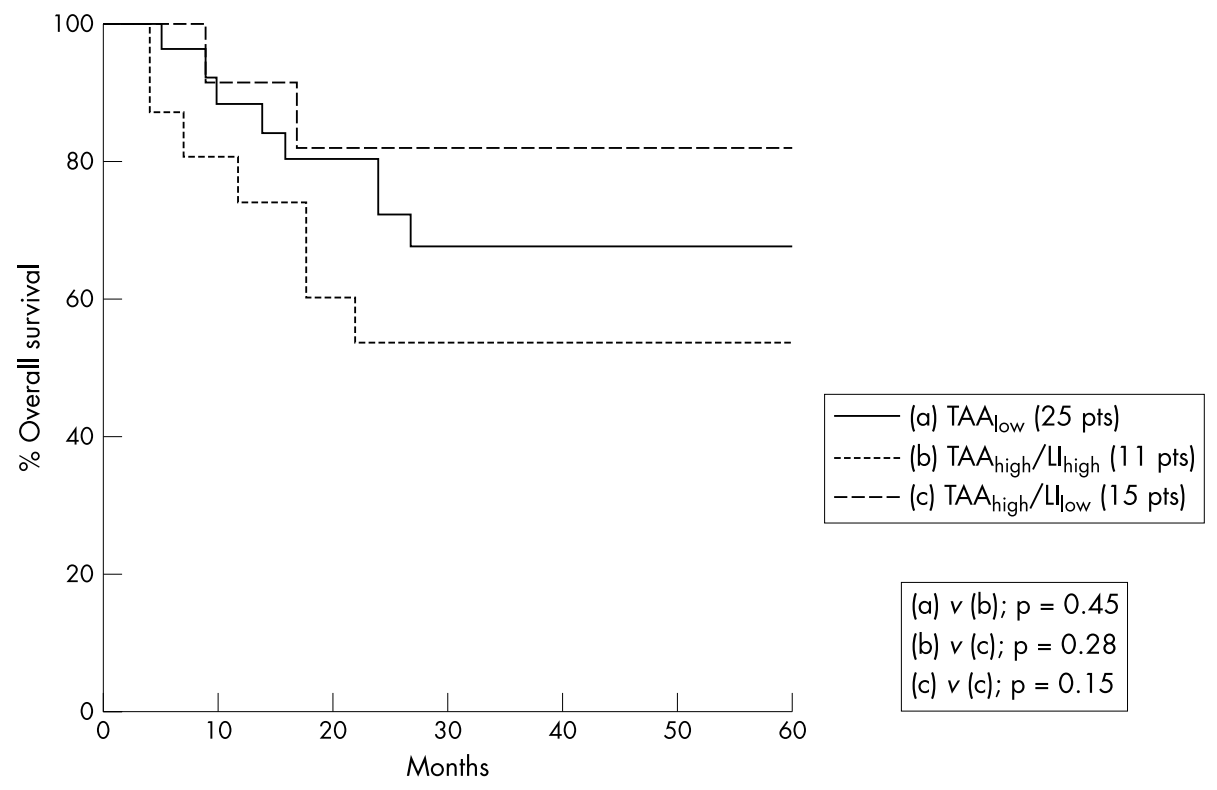

Figure 4 Kaplan-Meier survival curves with double stratification for tumour angiogenic activity (TAA) and the degree of the lymphocytic infiltration (LI). Note that worse prognosis was confirmed for highly angiogenic tumours only in the absence of a lymphocytic response. 


\section{Take home messages}

- Tumour angiogenic activity (TAA) and vascular survival ability (VSA) provide a more complete profile of the tumour vasculature and are associated with the aggressiveness of tumour behaviour in transitional cell carcinomas of the urinary bladder

- The inclusion of VSA as an additional parameter to TAA may be important for the identification of angiogenic tumours with differential responses to various antiangiogenic treatments

findings suggest that the ability of bladder cancer to maintain a viable vasculature in the inner tumour areas may be associated with aggressive tumour behaviour, whereas the escape of cancer cells to the lymphatics is dependent on the angiogenic process along the tumour-normal bladder interface (TAA). Similar suggestions have been put forward after analysis performed in lung carcinomas. ${ }^{7}$ A high VSA was also directly related to high tumour cell PI, and marginally to limited necrosis. Therefore, it appears that VSA is a more informative marker than TAA. In fact, survival analysis showed that high VSA is an important marker of poor prognosis. It seems that although TAA facilitates cancer cell escape and metastasis to lymph nodes and distant organs, it is the VSA that predicts an increased ability of tumour cells to establish viable tumours in metastatic sites once the tumour cells have reached the host's stroma through the circulation. Similar findings have been reported previously by our group in lung, colon, and breast carcinomas. ${ }^{7-9}$

"The ability of bladder cancer to maintain a viable vasculature in the inner tumour areas may be associated with aggressive tumour behaviour"

Thus, we conclude that the growth and survival of the newly formed vascular network of tumours may be controlled, at least in part, by different molecular pathways beyond TAA. Assessment of VSA, in parallel with TAA, provides a better profile of the quality of the tumour vasculature and is associated with the aggressiveness of tumour behaviour in transitional cell carcinomas of the urinary bladder. The inclusion of VSA as an additional parameter to TAA for the characterisation of the bladder cancer vasculature may prove of importance for the identification of subgroups of angiogenic tumours with differential responsiveness to the plethora of antiangiogenic and angiotoxic agents currently under preclinical and clinical evaluation.

\section{Authors' affiliations}

I Papadopoulos, Department of Urology, Democritus University of Thrace, Alexandroupolis, Greece

A Giatromanolaki, E Sivridis, Department of Pathology, Democritus University of Thrace

M I Koukourakis, Department of Radiotherapy/Oncology, Democritus University of Thrace

\section{REFERENCES}

1 Folkman J. Vascularisation of tumors. Sci Am 1976;234:58-73.

2 Sivridis E, Giatromanolaki A, Koukourakis MI. The vascular network of tumours. What is it not for? J Pathol 2003;201:173-80.

3 Fox SB, Gasparini G, Harris AL. Angiogenesis: pathological, prognostic, and growth-factor pathways and their link to trial design and anticancer drugs. Lancet Oncol 2001;2:278-89.

4 Harris AL. Hypoxia: a key regulatory factor in tumour growth. Nat Rev Cancer 2002;2:38-47.

5 Thompson WD, Shiach KJ, Fraser RA, et al. Tumours acquire their vasculature by vessel incorporation, not vessel ingrowth. J Pathol 1987;151:323-32.

6 Koukourakis MI, Giatromanolaki A, Sivridis E, et al. Cancer vascularization implications in radiotherapy? Int J Radiat Oncol Biol Phys 2000;48:545-53.

7 Giatromanolaki A, Koukourakis MI, Sivridis E, et al. "Invading edge vs. inner" (edvin) patterns of vascularization: an interplay between angiogenic and vascular survival factors defines the clinical behaviour of non-small cell lung cancer. J Pathol 2000;192:140-9.

8 Giatromanolaki A, Sivridis E, Minopoulos G, et al. Differential assessment of vascular survival ability and tumor angiogenic activity in colorectal cancer. Clin Cancer Res 2002;8:1185-91.

9 Giatromanolaki A, Sivridis E, Simopoulos C, et al. Differential assessment of angiogenic activity and of vascular survival ability (VSA) in breast cancer. Clin Exp Metastasis 2002;19:673-9.

10 Streeter EH, Crew JP. Angiogenesis, angiogenic factor expression and prognosis of bladder cancer. Anticancer Res 2001;21:4355-63.

11 Dickinson AJ, Fox SB, Persad RA, et al. Quantification of angiogenesis as an independent predictor of prognosis in invasive bladder carcinomas. $\mathrm{Br} J$ Urol 1994;74:762-6.

12 Bochner BH, Cote RJ, Weidner N, et al. Angiogenesis in bladder cancer: relationship between microvessel density and tumor prognosis. J Natl Cancer Inst 1995:87:1603-12.

13 Philp EA, Stephenson TJ, Reed MW. Prognostic significance of angiogenesis in transitional cell carcinoma of the human urinary bladder. $\mathrm{Br} J$ Urol 1996;77:352-7.

14 Chaudhary R, Bromley M, Clarke NW, et al. Prognostic relevance of microvessel density in cancer of the urinary bladder. Anticancer Res 1999; 19:3479-84.

15 Inoue K, Slaton JW, Karashima T, et al. The prognostic value of angiogenesis factor expression for predicting recurrence and metastasis of bladder cancer after neoadjuvant chemotherapy and radical cystectomy. Clin Cancer Res 2000:6:4866-73.

16 Korkolopoulou P, Konstantinidou AE, Kavantzas N, et al. Morphometric microvascular characteristics predict prognosis in superficial and invasive bladder cancer. Virchows Arch 2001;438:603-11.

17 Elsobky E, El-Baz M, Gomha M, et al. Prognostic value of angiogenesis in schistosoma-associated squamous cell carcinoma of the urinary bladder. Urology 2000;60:69-73.

18 Offersen BV, Knap MM, Marcussen N, et al. Intense inflammation in bladder carcinoma is associated with angiogenesis and indicates good prognosis. Br J Cancer 2000;87:1422-30.

19 Sagol O, Yorukoglu K, Sis B, et al. Does angiogenesis predict recurrence in superficial transitional cell carcinoma of the bladder? Urology 2001;57:895-9.

20 Giatromanolaki A, Koukourakis M, Theodossiou D, et al Comparative evaluation of angiogenesis assessment with anti-factor VIII and anti-CD31 immunostaining in non small cell lung cancer. Clin Cancer Res 1997;3:2485-92.

21 Giatromanolaki A, Stathopoulos GP, Tsiobanou E, et al. Combined role of tumor angiogenesis, bcl-2, and p53 expression in the prognosis of patients with colorectal carcinoma. Cancer 1999;86:1421-30. 